\title{
Higgs couplings and Naturalness in the littlest Higgs model with T-parity at the LHC and TLEP
}

\author{
Bingfang Yang, ${ }^{a, b}$ Guofa $\mathbf{M i}^{a}$ and Ning Liu $^{b}$ \\ ${ }^{a}$ School of Materials Science and Engineering, Henan Polytechnic University, \\ Jiaozuo 454000, China \\ ${ }^{b}$ Institute of Theoretical Physics, Henan Normal University, \\ Xinxiang 453007, China \\ E-mail: yangbingfang@gmail.com, peter@hpu.edu.cn, \\ wlln@mail.ustc.edu.cn
}

ABSTRACT: Motivated by the recent LHC Higgs data and null results in searches for any new physics, we investigate the Higgs couplings and naturalness in the littlest Higgs model with T-parity. By performing the global fit of the latest Higgs data, electroweak precise observables and $R_{b}$ measurements, we find that the scale $f$ can be excluded up to $600 \mathrm{GeV}$ at $2 \sigma$ confidence level. The expected Higgs coupling measurements at the future collider TLEP will improve this lower limit to above $3 \mathrm{TeV}$. Besides, the top parnter mass $m_{T_{+}}$can be excluded up to $880 \mathrm{GeV}$ at $2 \sigma$ confidence level. The future HL-LHC can constrain this mass in the region $m_{T_{+}}<2.2 \mathrm{TeV}$ corresponding to the fine-tuning being lager than $1 \%$.

Keywords: Hadronic Colliders, Phenomenological Models

ARXIV EPRINT: 1407.6123 


\section{Contents}

1 Introduction 1

2 A brief review of the LHT model 2

3 Calculations and numerical results 4

4 Conclusions $\quad 10$

\section{Introduction}

The discovery of a Higgs boson [1-4] by the ATLAS [5] and CMS [6] collaborations at the LHC marks a milestone of an effort that has been ongoing for almost half a century and opens up a new era of particle physics. The existing measurements [7-10] and the global fits to the ATLAS and CMS Higgs data within remarkable precision [11-20] agree with the standard model (SM) predictions. This conclusion is consistent with the ATLAS and CMS null results in searches for any new physics. However, the experiments of cold dark matter [21] and neutrino oscillations [22] cannot be explained in the framework of the SM so that they are supposed to provide obvious evidence for the new physics beyond the SM. In particular, the facts that the SM can be an effective theory valid all the way up to the Planck scale and there is no symmetry protecting the scalar masses lead to the naturalness problem, i.e., why the Higgs boson mass is of the order of the electroweak scale and not driven by the radiative corrections to the Planck scale, remains unanswered.

Since the discovery of the Higgs boson the fine-tuning problem has become even more intriguing. Among many new physics models, Little Higgs models based on a collective symmetry breaking can provide a natural explanation of the fine-tuning by constructing the Higgs as a pseudo-goldstone boson. The littlest Higgs (LH) model [23-26] is an economical approach to implement the idea of the little Higgs theory. However, due to the large corrections to the electroweak precision observables (EWPO) from the mixing of the SM gauge bosons and the heavy gauge bosons, the original LH model is severely constrained by precision electroweak data. This constraint can be relaxed by introducing the discrete symmetry T-parity, which is dubbed as littlest Higgs model with T-parity(LHT) [27-30].

With current data, all properties of the observed Higgs-like particle turn out to be in rough agreement with expectations of the SM [31-35], but there are still some rooms for the new physics [36, 37], which may be ultimately examined at the LHC-Run2 and the future Higgs factories [38-49]. Since top partner is naturally related to the Higgs physics and plays an important role in the naturalness problem, one can obtain constraints from the Higgs data [50-55]. In this work, we will discuss the Higgs couplings and the naturalness problem in the LHT model at the LHC and Triple-Large Electron-Positron Collider (TLEP) [56, 57] by performing a global fit of the latest Higgs data, $R_{b}$ and oblique parameters, and give the current and future constraints to the LHT parameters. 
Recently, some similar works have been carried out in refs. [58-61]. Different from these papers, we perform a state-of-the-art global fit to obtain the indirect constraints on the breaking scale and the top partner with a comprehensive way. This method was widely used in the fit of the SM to the electroweak precision data. So, it will be also meaningful to explore what might happen in the LHT model with a global fit at future colliders. By building an overall likelihood function for the constraints from the EWPO, $R_{b}$ measurements and Higgs data, we can obtain a well-defined statistical results of the exclusion limit on the breaking scale. More importantly, we obtain the exclusion limit on the top partner mass, which is obvious absent in other papers.

This paper is organized as follows. In section 2, we give a brief description of the LHT model. In section 3, we present the calculation methodology and the numerical results at the LHC and the TLEP. Finally, we draw our conclusions in section 4.

\section{A brief review of the LHT model}

The LHT model is a non-linear $\sigma$ model based on the coset space $\mathrm{SU}(5) / \mathrm{SO}(5)$, where the spontaneous symmetry breaking is realised at the scale $f$ via the vacuum expectation value (VEV) of an $\mathrm{SU}(5)$ symmetric tensor $\Sigma$, given by

$$
\Sigma_{0}=\langle\Sigma\rangle\left(\begin{array}{ccc}
\mathbf{0}_{2 \times 2} & 0 & \mathbf{1}_{2 \times 2} \\
0 & 1 & 0 \\
\mathbf{1}_{2 \times 2} & 0 & \mathbf{0}_{2 \times 2}
\end{array}\right) .
$$

The VEV of $\Sigma_{0}$ breaks the gauged subgroup $[\mathrm{SU}(2) \times \mathrm{U}(1)]^{2}$ of $\mathrm{SU}(5)$ down to the SM electroweak $\mathrm{SU}(2)_{L} \times \mathrm{U}(1)_{Y}$, which leads to new heavy gauge bosons $W_{H}^{ \pm}, Z_{H}, A_{H}$. After the EWSB, their masses up to $\mathcal{O}\left(v^{2} / f^{2}\right)$ are given by

$$
M_{W_{H}}=M_{Z_{H}}=g f\left(1-\frac{v^{2}}{8 f^{2}}\right), \quad M_{A_{H}}=\frac{g^{\prime} f}{\sqrt{5}}\left(1-\frac{5 v^{2}}{8 f^{2}}\right)
$$

with $g$ and $g^{\prime}$ being the SM SU(2) and U(1) gauge couplings, respectively. In order to match the SM prediction for the gauge boson masses, the VEV $v$ needs to be redefined via the functional form

$$
v=\frac{f}{\sqrt{2}} \arccos \left(1-\frac{v_{\mathrm{SM}}^{2}}{f^{2}}\right) \simeq v_{\mathrm{SM}}\left(1+\frac{1}{12} \frac{v_{\mathrm{SM}}^{2}}{f^{2}}\right),
$$

where $v_{\mathrm{SM}}=246 \mathrm{GeV}$ is the SM Higgs VEV.

Under the unbroken $\mathrm{SU}(2)_{L} \times \mathrm{U}(1)_{Y}$ the Goldstone boson matrix $\Pi$ is given by

$$
\Pi=\left(\begin{array}{ccc}
0 & \frac{H}{\sqrt{2}} & \Phi \\
\frac{H^{\dagger}}{\sqrt{2}} & 0 & \frac{H^{T}}{\sqrt{2}} \\
\Phi^{\dagger} & \frac{H^{*}}{\sqrt{2}} & 0
\end{array}\right),
$$

where $H$ is the little Higgs doublet $\left(h^{+}, h\right)^{T}$ and $\Phi$ is a complex triplet under $\mathrm{SU}(2)_{L}$ which forms a symmetric tensor

$$
\Phi=\frac{-i}{\sqrt{2}}\left(\begin{array}{cc}
\sqrt{2} \phi^{++} & \phi^{+} \\
\phi^{+} & \phi^{0}+i \phi^{P}
\end{array}\right) .
$$


$\phi^{0}$ and $\phi^{P}$ are both real scalars, whereas the $\phi^{++}$and $\phi^{+}$are complex scalars. The other Goldstone bosons are the longitudinal modes of the heavy gauge bosons and therefore will not appear in unitary gauge. The mass of $\Phi$ can be given by

$$
m_{\Phi}=\frac{2 m_{H} f}{v},
$$

where all components of the triplet are degenerate at the order we are examining.

When T-parity is implemented in the quark sector of the model, we require the existence of mirror partners with T-odd quantum number for each SM quark. We denote the up and down-type mirror quarks by $u_{H}^{i}$ and $d_{H}^{i}$, where $i(i=1,2,3)$ is the generation index. After the EWSB, their masses up to $\mathcal{O}\left(v^{2} / f^{2}\right)$ are given by

$$
m_{d_{H}^{i}}=\sqrt{2} \kappa_{i} f, \quad m_{u_{H}^{i}}=m_{d_{H}^{i}}\left(1-\frac{v^{2}}{8 f^{2}}\right)
$$

where $\kappa_{i}$ are the diagonalized Yukawa couplings of the mirror quarks. One can notice that the down-type mirror quarks have no interactions with the Higgs.

In order to stabilize the Higgs mass, an additional T-even heavy quark $T_{+}$is introduced to cancel the large one-loop quadratic divergences caused by the top quark. Meanwhile, the implementation of T-parity requires a T-odd mirror partner $T_{-}$with $T_{+}$. The T-even quark $T_{+}$mix with the SM top-quark and leads to a modification of the top quark couplings relatively to the SM. The mixing can be parameterized by dimensionless ratio $R=\lambda_{1} / \lambda_{2}$, where $\lambda_{1}$ and $\lambda_{2}$ are two dimensionless top quark Yukawa couplings. This mixing parameter can also be used by $x_{L}$ with

$$
x_{L}=\frac{R^{2}}{1+R^{2}} .
$$

Considering only the largest corrections induced by EWSB, their masses up to $\mathcal{O}\left(v^{2} / f^{2}\right)$ are then given by

$$
\begin{aligned}
m_{t} & =\lambda_{2} \sqrt{x_{L}} v\left[1+\frac{v^{2}}{f^{2}}\left(-\frac{1}{3}+\frac{1}{2} x_{L}\left(1-x_{L}\right)\right)\right] \\
m_{T_{+}} & =\frac{f}{v} \frac{m_{t}}{\sqrt{x_{L}\left(1-x_{L}\right)}}\left[1+\frac{v^{2}}{f^{2}}\left(\frac{1}{3}-x_{L}\left(1-x_{L}\right)\right)\right] \\
m_{T_{-}} & =\frac{f}{v} \frac{m_{t}}{\sqrt{x_{L}}}\left[1+\frac{v^{2}}{f^{2}}\left(\frac{1}{3}-\frac{1}{2} x_{L}\left(1-x_{L}\right)\right)\right] .
\end{aligned}
$$

The corrections to the Higgs couplings of the other two generations of T-even (SM-like) up-type quarks up to $\mathcal{O}\left(v_{\mathrm{SM}}^{4} / f^{4}\right)$ are given by

$$
\frac{g_{h \bar{u} u}}{g_{h \bar{u} u}^{\mathrm{SM}}}=1-\frac{3}{4} \frac{v_{\mathrm{SM}}^{2}}{f^{2}}-\frac{5}{32} \frac{v_{\mathrm{SM}}^{4}}{f^{4}} \quad u \equiv u, c .
$$

For the T-even (SM-like) down-type quarks and charged leptons, the Yukawa interaction have two possible constructions [62]. The corresponding corrections to the Higgs couplings with respect to their SM values up to $\mathcal{O}\left(v_{\mathrm{SM}}^{4} / f^{4}\right)$ are given by $\left(d \equiv d, s, b, l_{i}^{ \pm}\right)$

$$
\begin{array}{ll}
\frac{g_{h \bar{d} d}}{g_{h \bar{d} d}^{\mathrm{SM}}}=1-\frac{1}{4} \frac{v_{\mathrm{SM}}^{2}}{f^{2}}+\frac{7}{32} \frac{v_{\mathrm{SM}}^{4}}{f^{4}} & \text { Case A } \\
\frac{g_{h \bar{d} d}}{g_{h \bar{d} d}^{\mathrm{SM}}}=1-\frac{5}{4} \frac{v_{\mathrm{SM}}^{2}}{f^{2}}-\frac{17}{32} \frac{v_{\mathrm{SM}}^{4}}{f^{4}} & \text { Case B. }
\end{array}
$$


One can notice that Case B predicts a stronger suppression for the down-type fermion couplings to the Higgs boson.

The naturalness of the model can be quantified by how much the contributions from the heavy states $\left(\delta \mu^{2}\right)$ exceed the observed value of the Higgs mass squared parameter $\left(\mu_{\text {obs }}^{2}\right)[23]$ :

$$
\Delta=\frac{\left|\delta \mu^{2}\right|}{\mu_{\mathrm{obs}}^{2}}, \quad \mu_{\mathrm{obs}}^{2}=\frac{m_{h}^{2}}{2} .
$$

Here $m_{h}$ is the Higgs boson mass. In the LHT model, the dominant negative log-divergent contribution to the Higgs mass squared parameter comes from the top quark and its heavy partner $T_{+}$loops [23]

$$
\delta \mu^{2}=-\frac{3 \lambda_{t}^{2} m_{T_{+}}^{2}}{8 \pi^{2}} \log \frac{\Lambda^{2}}{m_{T_{+}}^{2}}
$$

where $\Lambda=4 \pi f$ is the cut-off of the nonlinear sigma model, $\lambda_{t}$ is the SM top Yukawa coupling and $m_{T_{+}}$is the mass of the heavy top partner.

\section{Calculations and numerical results}

In our numerical calculations, we take the SM input parameters as follows [63]:

$$
m_{t}=173.5 \mathrm{GeV}, \quad m_{W}=80.385 \mathrm{GeV}, \quad \alpha\left(m_{Z}\right)=1 / 127.918, \quad \sin ^{2} \theta_{W}=0.231 .
$$

Our global fit is based on the frequentist theory. For a set of observables $\mathcal{O}_{i}(i=$ $1 \ldots N)$, the experimental measurements are assumed to be Gaussian distributed with the mean value $\mathcal{O}_{i}^{\exp }$ and error $\sigma_{i}^{\exp }$. The $\chi^{2}$ can be defined as $\chi^{2}=\sum_{i}^{N} \frac{\left(\mathcal{O}_{i}^{\text {th }}-\mathcal{O}_{i}^{\exp }\right)^{2}}{\sigma_{i}{ }^{2}}$, where $\sigma_{i}$ is the total error both experimental and theoretical. The likelihood $\mathcal{L} \equiv \exp \left[-\sum \chi_{i}^{2}\right]$ for a point in the parameter space is calculated by using the $\chi^{2}$ statistics as a sum of individual contributions from the latest experimental constraints. The confidence regions are evaluated with the profile-likelihood method from tabulated values of $\delta \chi^{2} \equiv-2 \ln \left(\mathcal{L} / \mathcal{L}_{\text {max }}\right)$. In three dimensions, $68.3 \%$ confidence regions (corresponding to $1 \sigma$ range) are given by $\delta \chi^{2}=$ 3.53 and $95.0 \%$ confidence regions (corresponding to $2 \sigma$ range) are given by $\delta \chi^{2}=8.02$.

Under few assumptions involving mainly flavour independence in the mirror fermion sector, the LHT model can be parametrised by only three free parameters, i.e., the scale $f$, the ratio $R$ and the Yukawa couplings of the mirror quarks $\kappa_{j}$. Considering the recent constraint from the searches for the monojet, we require the lower bound on the Yukawa couplings of the mirror quarks are $\kappa_{j} \geq 0.6$ [61]. We scan over these parameters within the following ranges [58-61, 64]

$$
500 \mathrm{GeV} \leq f \leq 2000 \mathrm{GeV}, \quad 0.1 \leq R \leq 3.3, \quad 0.6 \leq \kappa_{j} \leq 3,
$$

where we assume the three generations $\kappa_{j}$ are degenerate. The couplings of the UV operators are set as $c_{s}=c_{t}=1$. The likelihood function $\mathcal{L}$ is constructed from the following constraints:

1. EWPO: these oblique corrections can be described in terms of the Peskin-Takeuchi S, $\mathrm{T}$ and $\mathrm{U}$ parameters [65]. Firstly, the top partner can contribute to the propagators of the electroweak gauge bosons at one-loop level. In contrast to $T_{+}$, the T-odd top partner $T_{-}$does not contribute to $\mathrm{S}, \mathrm{T}, \mathrm{U}$ parameters since it is an $\mathrm{SU}(2)_{L}$ singlet 
which does not mix with the SM top quark. Secondly, the T-odd mirror fermions give a contribution to the $\mathrm{T}$ parameter at one-loop, which can have a noticeable effect on the EWPO due to a large number (twelve) of doublets in the SM; thirdly, another important correction to both the $\mathrm{S}$ and $\mathrm{T}$ parameters follows from the modified couplings of the Higgs boson to the SM gauge bosons. Finally, other possible contributions arise from new operators which parametrize the effects of the UV physics on weak scale observables. All these different contributions to the oblique parameters should be summed up. We calculate $\chi^{2}$ by using the formulae in refs. [64, 66, 67] and adopting the experimental values of $\mathrm{S}, \mathrm{T}$ and $\mathrm{U}$ in the ref. [63].

2. $R_{b}$. The branching ratio $R_{b}$ is very sensitive to the new physics beyond the SM, the precision experimental value of $R_{b}$ may give a severe constraint on the new physics. In the LHT model, there are new fermions and new gauge bosons, which can contribute to the $Z b \bar{b}$ coupling and give corrections to the $R_{b}$ at one-loop level [68]. The final combined result from the LEP and SLD measurements show $R_{b}=0.21629 \pm$ 0.00066 [63], which is consistent with the $\mathrm{SM}$ prediction $R_{b}^{\mathrm{SM}}=0.21578_{-0.0008}^{+0.0005}$.

3. Higgs data. The experimental results are given in terms of signal strengths $\mu(X ; Y)$, which is defined as the ratio of the observed rate for Higgs process $X \rightarrow h \rightarrow$ $Y$ relative to the prediction for the SM Higgs, $\mu(X ; Y) \equiv \frac{\sigma(X) B R(h \rightarrow Y)}{\sigma\left(X_{\mathrm{SM}}\right) B R\left(h_{\mathrm{SM}} \rightarrow Y\right)}$. We confront the modified Higgs interactions and the one-loop contribution of the new particles in the LHT model with the available Higgs data. We calculate the $\chi^{2}$ values by using the public package HiggsSignals-1.2.0 [69, 70], which includes 81 channels from the LHC and Tevatron and these experimental data are listed in ref. [71]. In our calculations, the Higgs mass $m_{h}$ is fixed as $126 \mathrm{GeV}$. Note that for the Higgs data, the HiggsSignals has provided the calculation of $\chi^{2}$, where both experimental (systematic and statistical) uncertainties as well as SM theory uncertainties are included.

In figure 1, we show the results of the global fit to the above three kinds of constraints in the plane of $R$ versus $f$ for Case A and Case B, respectively. We can see that the lower bound on the symmetry breaking scale at $95 \%$ C.L. is

$$
\begin{array}{ll}
f>670 \mathrm{GeV} & \text { Case A, } \\
f>600 \mathrm{GeV} & \text { Case B. }
\end{array}
$$

The constraints are stronger than the electroweak precision constraints in ref. [64], which is because the main constraint here comes from the Higgs data. For the top partner mass, we can see that the combined indirect constraints can exclude $m_{T_{+}}$at $95 \%$ C.L. up to

$$
\begin{array}{ll}
m_{T_{+}}>980 \mathrm{GeV} & \text { Case A, } \\
m_{T_{+}}>880 \mathrm{GeV} & \text { Case B. }
\end{array}
$$

It's worth noting that they are stronger than the lower bound set by the ATLAS direct searches for the $\mathrm{SU}(2)$ singlet top partner, $m_{T}>640 \mathrm{GeV}$ [72]. Our study may play a complementary role to the direct searches in probing top partner. 

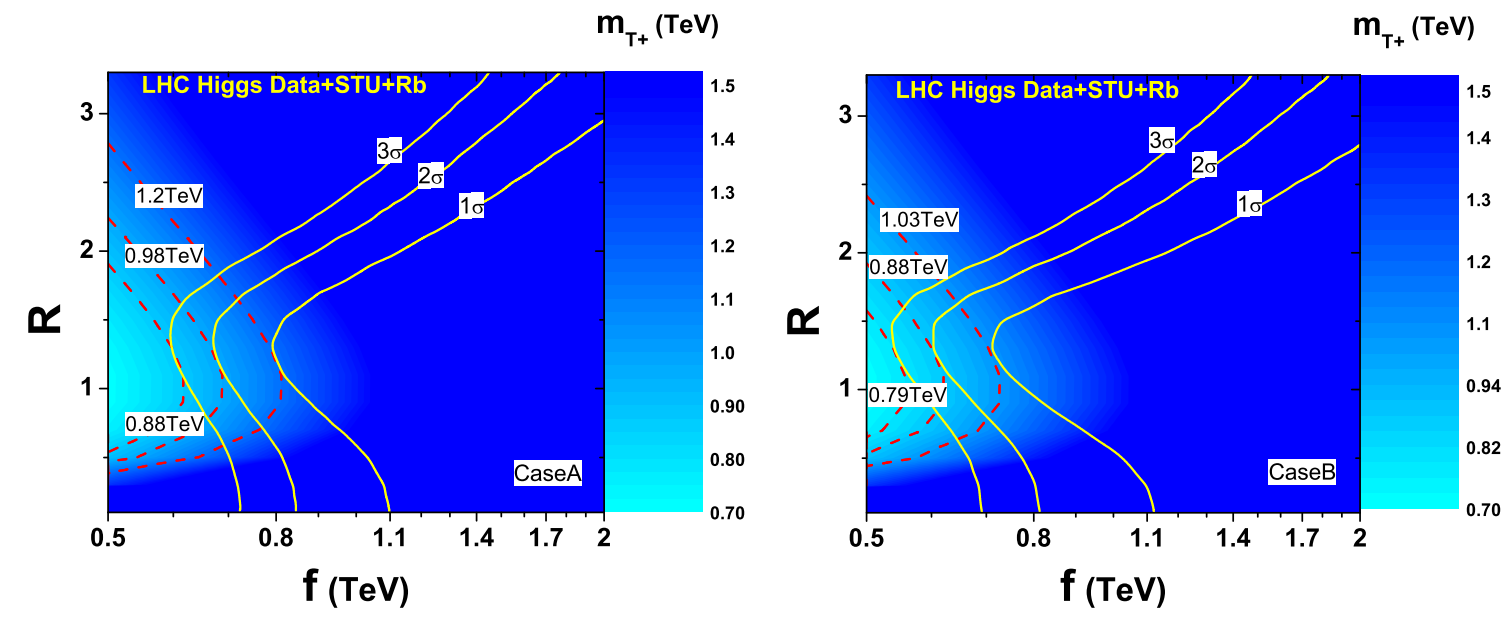

Figure 1. The global fit of the constraints on the LHT model in the $R-f$ plane for Case A and Case B. The yellow lines from right to left respectively correspond to $1 \sigma, 2 \sigma$ and $3 \sigma$ exclusion limits.

\begin{tabular}{|c|c|c|}
\hline Facility & HL-LHC & TLEP \\
\hline$\sqrt{s}$ & $14 \mathrm{TeV}$ & $240 \mathrm{GeV}$ \\
$\int \mathcal{L} d t$ & $3000\left(\mathrm{fb}^{-1}\right)$ & $10000\left(\mathrm{fb}^{-1}\right)$ \\
\hline$\kappa_{\gamma}$ & $2-5 \%$ & $1.7 \%$ \\
$\kappa_{g}$ & $3-5 \%$ & $1.1 \%$ \\
$\kappa_{W}$ & $2-5 \%$ & $0.85 \%$ \\
$\kappa_{Z}$ & $2-4 \%$ & $0.16 \%$ \\
\hline$\kappa_{u}$ & $7-10 \%$ & - \\
$\kappa_{d}$ & $4-7 \%$ & - \\
$\kappa_{c}$ & $7-10 \%$ & $1.0 \%$ \\
$\kappa_{s}$ & $4-7 \%$ & - \\
$\kappa_{t}$ & $7-10 \%$ & - \\
$\kappa_{b}$ & $4-7 \%$ & $0.88 \%$ \\
\hline
\end{tabular}

Table 1. Expected precision on the Higgs couplings to quarks and vector bosons at the HL-LHC and the TLEP.

The expected precision for the Large Hadron Collider High-Luminosity Upgrade (HLLHC) and the TLEP are assumed in table 1, which comes from the table 14 and table 16 of the Higgs working group report [73].

In the LHT model, the loop-induced couplings $h g g$ and $h \gamma \gamma$ can receive contributions from both the modified couplings and the new particles. The decay $h \rightarrow g g$ can be corrected by the modified $h t \bar{t}$ coupling and the loops of top partner $T_{+}$and T-odd mirror quarks. In addition to these corrections involved in the decay $h \rightarrow g g$, the decay $h \rightarrow \gamma \gamma$ can be also corrected by the modified $h W W$ coupling and the loops of $W_{H}, \phi^{+}, \phi^{++}$. Besides, the couplings $h c \bar{c}, h s \bar{s}, h b \bar{b}, h Z Z$ are also modified, they can exert an effect on our fit.

In figure 2 and figure 3, we show the shifts of the Higgs couplings $h V V, h t \bar{t}, h g g, h \gamma \gamma$ for the above samples in the $2 \sigma$ range. In order to investigate the observability, we compare 

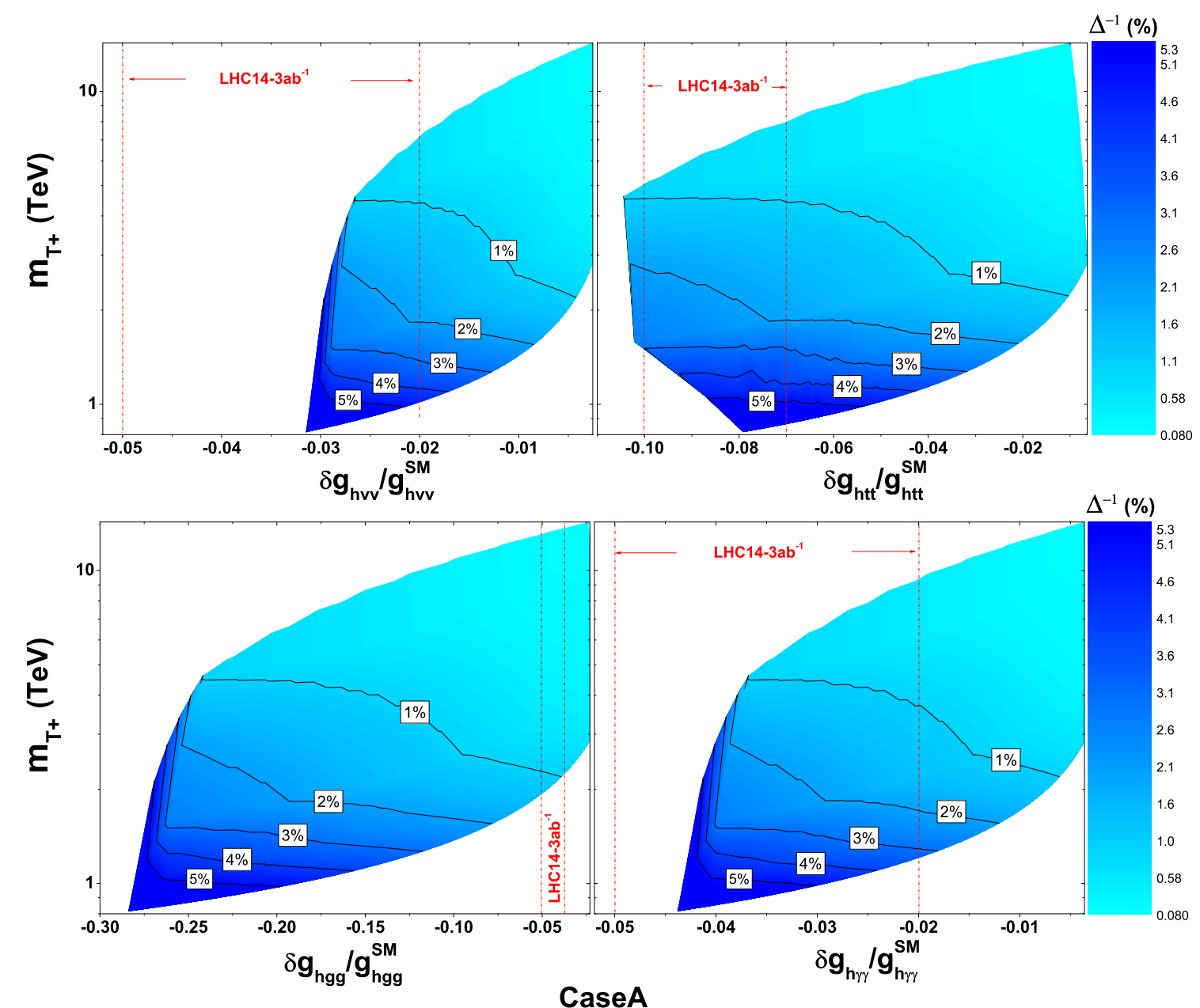

Figure 2. The shifts of the Higgs couplings for the samples in the $2 \sigma$ allowed range in figure 1 for Case A. The red dash-dot lines represent the expected measurement uncertainties at HL-LHC.

them with the corresponding expected measurement uncertainties of the Higgs couplings in table 1 at HL-LHC with a luminosity of $3000 \mathrm{fb}^{-1}$. The value of the fine-tuning for each point is also calculated by using the eq. (2.14). From figure 2 and figure 3, we can have some observations as follows:

1. The values of the fine-tuning for the samples are cornered to be smaller than about $6 \%$ by the above global fit.

2. For the Higgs couplings $h V V$ and $h t \bar{t}$, they are suppressed by the high order factor $\mathcal{O}\left(v^{2} / f^{2}\right)$. The deviation of the Higgs couplings $g_{h V V}$ from the SM predictions are at percent level and the deviation of the Higgs coupling $g_{h t \bar{t}}$ from the SM prediction can reach over $10 \%$.

For the loop-induced couplings $g_{h g g}$ and $g_{h \gamma \gamma}$, on one hand they are corrected by the high order factor, on the other hand they are corrected by the loop contributions of the new particles. For the effects of these loop diagrams, there are cancelation 

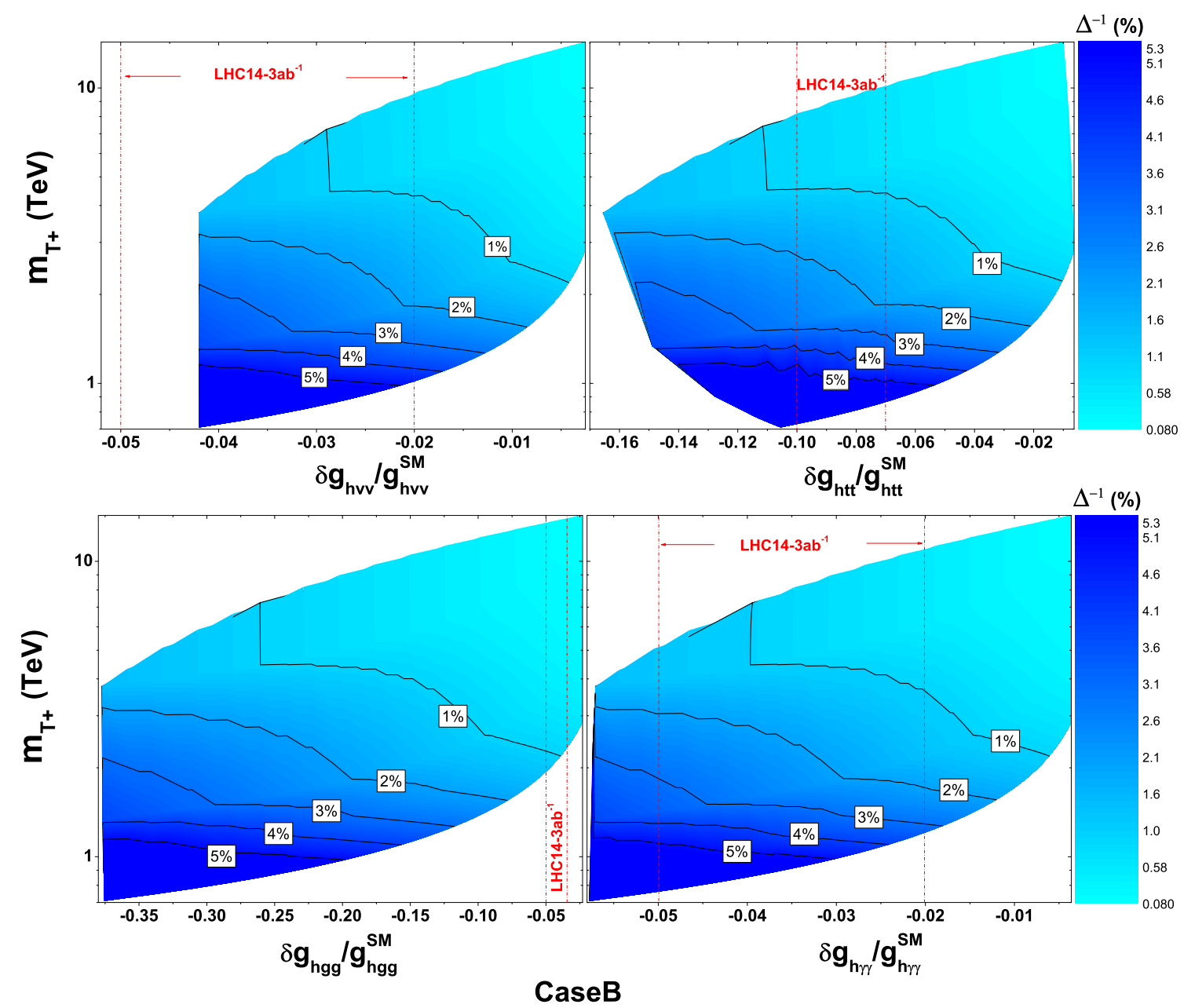

Figure 3. The shifts of the Higgs couplings for the samples in the $2 \sigma$ allowed range in figure 1 for Case B. The red dash-dot lines represent the expected measurement uncertainties at HL-LHC.

between $t\left(W_{L}\right)$ and the corresponding partner $T_{+}\left(W_{H}\right)$ so that the effective $g_{h g g}$ and $g_{h \gamma \gamma}$ couplings are reduced. The deviation of the Higgs coupling $g_{h \gamma \gamma}$ from the SM prediction is at percent level, that is because the dominant contribution to the coupling $g_{h \gamma \gamma}$ comes from the $W_{L}\left(W_{H}\right)$ over the $t\left(T_{+}\right)$. The Higgs coupling $g_{h g g}$ from the SM prediction can reach about $30 \%$, that is because the dominant contribution to the coupling $g_{h g g}$ comes from the $h t \bar{t}$ coupling and $t\left(T_{+}\right)$loops, where the contribution of $h t \bar{t}$ coupling accounts for about $10 \%$ and the contributions of $t\left(T_{+}\right)$loops account for about 20\%. Furthermore, we can see that the deviations for Case A are less than that for Case B, which originate from the stronger suppression for the down-type fermion couplings to the Higgs boson in Case B.

Furthermore, we can see that all changes of the Higgs couplings are negative. In the LHT model, in order to cancel the quadratic divergence of the Higgs mass, the heavy gauge bosons and the additional heavy quark $T_{+}$are introduced. This leads 

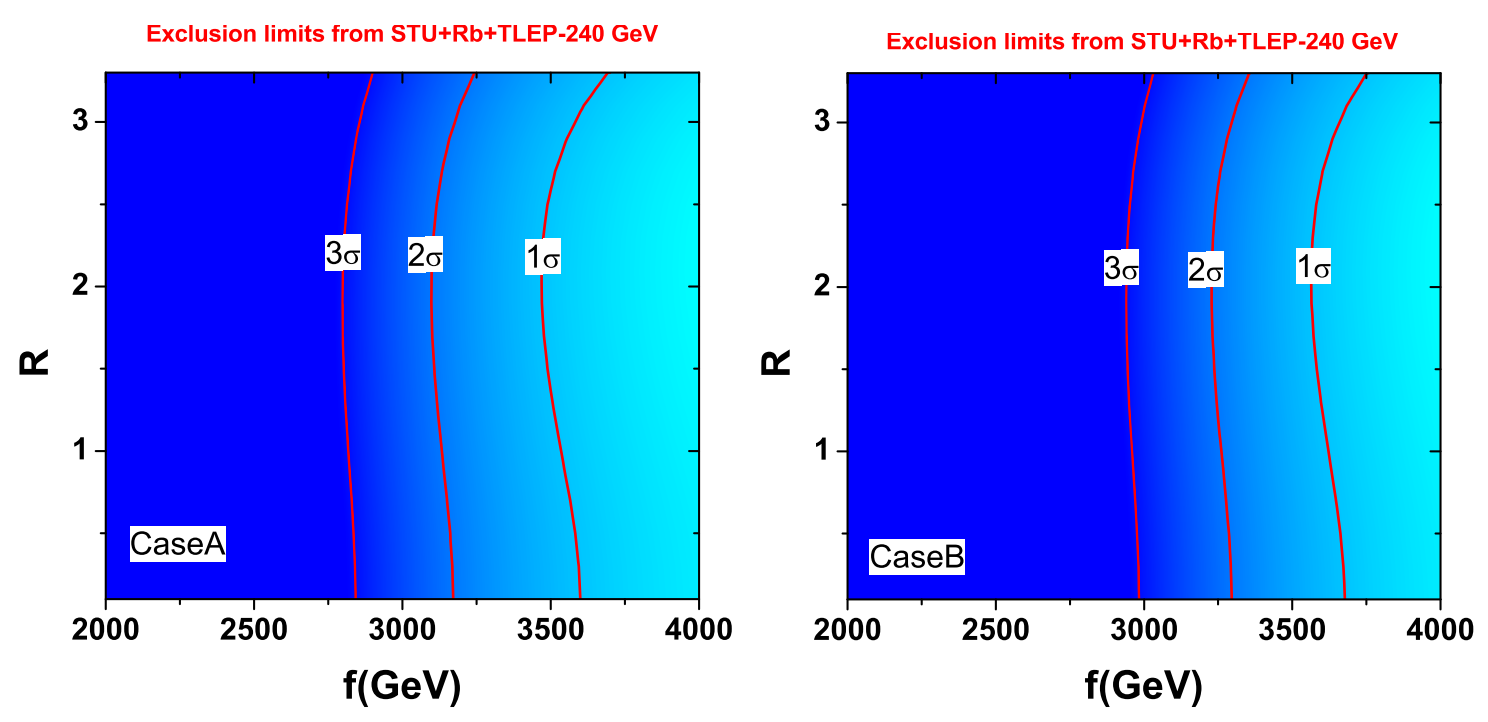

Figure 4. The expected exclusion limits on the $R-f$ plane for Case A and Case B from the global fit of EWPO, $R_{b}$ and TLEP.

to negative modification of the relevant couplings with respect to the SM. Besides, the non-linear expansion of the model field suppresses these couplings at the order $\mathcal{O}\left(v^{2} / f^{2}\right)$.

3. In figure 2 and figure 3 , we attempt to show the expected constraints from the future individual Higgs coupling meaurements on the top partner and naturalness at the HL-LHC. The couplings $h V V$ and $h t \bar{t}$ are modified at the order $\mathcal{O}\left(v^{2} / f^{2}\right)$, which can determine the scale $f$ and help us understand the nature of the Higgs boson in the LHT model. Apart from this, the coupling hgg can provide the information for the cancelation between $t$ and the corresponding partner $T_{+}$, while the coupling $h \gamma \gamma$ can provide the information for the cancelation between $W_{L}$ and the corresponding partner $W_{H}$. So, we can see that the individual Higgs coupling meaurements can help us understand the different parts of the LHT model.

4. The future measurements of the $g_{h g g}$ coupling at the HL-LHC will be able to exclude the $m_{T_{+}}<2.2 \mathrm{TeV}$, which corresponds to the fine-tuning being lager than about $1 \%$. However, other expected measurements, such as $g_{h V V}, g_{h t \bar{t}}$ and $g_{h \gamma \gamma}$ couplings, can only improve the limits for the top partner mass mildly.

In figure 4, we present the prospect of improving the constraints on the scale $f$ at a possible future Higgs factory TLEP with $\sqrt{s}=240 \mathrm{GeV}$. In our fit, the $\chi^{2}$ can be defined as

$$
\chi^{2}=\sum_{i}^{N} \frac{\left(\mu_{i}-1\right)^{2}}{\sigma_{i}{ }^{2}}
$$

where $\mu_{i}$ represents the signal strength prediction from the LHT model and $\sigma_{i}$ represents the $1 \sigma$ uncertainty i.e. the expected measurement precision at the TLEP. We use the Snowmass Higgs working group results to simply estimate the exclusion 
limits. Given that the super-high luminosity of $10000 \mathrm{fb}^{-1}$ can be achieved at the TLEP, we assume that all the measured Higgs couplings will be the same as the SM predictions with the expected measurement uncertainties in table 1. From the figure 4, we can see that the lower bound on the scale $f$ will be pushed up to $3.1 \mathrm{TeV}$ for Case A and 3.25 TeV for Case B at 95\% C.L..

\section{Conclusions}

In this paper, we investigated the Higgs couplings and naturalness in the LHT model under the available constraints from the current Higgs data and the EWPO. By performing the global fit, we find that the scale $f$ can be excluded up to $670 \mathrm{GeV}$ for Case A and $600 \mathrm{GeV}$ for Case B at $2 \sigma$ level. The precise measurements of the Higgs couplings at the future collider TLEP will constrain this limit to above $3 \mathrm{TeV}$. Besides, the top partner mass $m_{T_{+}}$ can be excluded up to $980 \mathrm{GeV}$ for Case A and $880 \mathrm{GeV}$ for Case B at $2 \sigma$ level. This mass can be constrained in the region $m_{T_{+}}<2.2 \mathrm{TeV}$ at the HL-LHC corresponding to the fine-tuning being lager than $1 \%$.

\section{Acknowledgments}

We would like to thank Marco Tonini for valuable discussions and also thank Lei Wu for helpful suggestions. This work was supported by the National Natural Science Foundation of China (NNSFC) under grants Nos. 11275245, 11305049, 11347140 and 11405047, by Specialized Research Fund for the Doctoral Program of Higher Education under Grant No.20134104120002, the Startup Foundation for Doctors of Henan Normal University under contract No.11112 and the Postdoctoral Science Foundation of China under Grant No. 2014M561987.

Open Access. This article is distributed under the terms of the Creative Commons Attribution License (CC-BY 4.0), which permits any use, distribution and reproduction in any medium, provided the original author(s) and source are credited.

\section{References}

[1] F. Englert and R. Brout, Broken Symmetry and the Mass of Gauge Vector Mesons, Phys. Rev. Lett. 13 (1964) 321 [INSPIRE].

[2] P.W. Higgs, Broken symmetries, massless particles and gauge fields, Phys. Lett. 12 (1964) 132 [inSPIRE].

[3] P.W. Higgs, Broken Symmetries and the Masses of Gauge Bosons, Phys. Rev. Lett. 13 (1964) 508 [INSPIRE].

[4] G.S. Guralnik, C.R. Hagen and T.W.B. Kibble, Global Conservation Laws and Massless Particles, Phys. Rev. Lett. 13 (1964) 585 [InSPIRE].

[5] ATLAS collaboration, Combined search for the Standard Model Higgs boson using up to $4.9 \mathrm{fb}^{-1}$ of pp collision data at $\sqrt{s}=7 \mathrm{TeV}$ with the ATLAS detector at the LHC, Phys. Lett. B 710 (2012) 49 [arXiv:1202.1408] [INSPIRE]. 
[6] CMS collaboration, Combined results of searches for the standard model Higgs boson in pp collisions at $\sqrt{s}=7 \mathrm{TeV}$, Phys. Lett. B 710 (2012) 26 [arXiv:1202.1488] [INSPIRE].

[7] ATLAS collaboration, Coupling properties of the new Higgs-like boson observed with the ATLAS detector at the LHC, ATLAS-CONF-2012-127 (2012).

[8] ATLAS collaboration, Updated coupling measurements of the Higgs boson with the ATLAS detector using up to $25 \mathrm{fb}^{-1}$ of proton-proton collision data, ATLAS-CONF-2014-009 (2014).

[9] CMS collaboration, Combination of standard model Higgs boson searches and measurements of the properties of the new boson with a mass near $125 \mathrm{GeV}$, CMS-PAS-HIG-12-045.

[10] CMS collaboration, Precise determination of the mass of the Higgs boson and studies of the compatibility of its couplings with the standard model, CMS-PAS-HIG-14-009.

[11] P.P. Giardino, K. Kannike, I. Masina, M. Raidal and A. Strumia, The universal Higgs fit, JHEP 05 (2014) 046 [arXiv:1303.3570] [INSPIRE].

[12] J. Ellis and T. You, Updated Global Analysis of Higgs Couplings, JHEP 06 (2013) 103 [arXiv: 1303.3879] [INSPIRE].

[13] A. Djouadi and G. Moreau, The couplings of the Higgs boson and its CP properties from fits of the signal strengths and their ratios at the $7+8 \mathrm{TeV} L H C$,

Eur. Phys. J. C 73 (2013) 2512 [arXiv:1303.6591] [INSPIRE].

[14] A. Falkowski, F. Riva and A. Urbano, Higgs at last, JHEP 11 (2013) 111 [arXiv:1303.1812] [INSPIRE].

[15] T. Alanne, S. Di Chiara and K. Tuominen, LHC Data and Aspects of New Physics, JHEP 01 (2014) 041 [arXiv:1303.3615] [INSPIRE].

[16] C. Englert et al., Precision Measurements of Higgs Couplings: Implications for New Physics Scales, J. Phys. G 41 (2014) 113001 [arXiv:1403.7191] [INSPIRE].

[17] J. Bernon, B. Dumont and S. Kraml, Status of Higgs couplings after Run-1 of the LHC using Lilith 1.0, arXiv:1409.1588 [INSPIRE].

[18] GFitter Group collaboration, M. Baak et al., The global electroweak fit at NNLO and prospects for the LHC and ILC, Eur. Phys. J. C 74 (2014) 3046 [arXiv:1407.3792] [INSPIRE].

[19] M.V. Marono, Future prospects of Higgs Physics at CMS, arXiv:1409.1711 [InSPIRE].

[20] J. Fan and M. Reece, A New Look at Higgs Constraints on Stops, JHEP 06 (2014) 031 [arXiv: 1401.7671] [INSPIRE].

[21] Planck collaboration, P.A.R. Ade et al., Planck 2013 results. XVI. Cosmological parameters, Astron. Astrophys. (2014) [arXiv:1303.5076] [INSPIRE].

[22] A. Strumia and F. Vissani, Neutrino masses and mixings and..., hep-ph/0606054 [INSPIRE].

[23] N. Arkani-Hamed, A.G. Cohen, E. Katz and A.E. Nelson, The Littlest Higgs, JHEP 07 (2002) 034 [hep-ph/0206021] [INSPIRE].

[24] S. Chang, A 'Littlest Higgs' model with custodial SU(2) symmetry, JHEP 12 (2003) 057 [hep-ph/0306034] [INSPIRE].

[25] T. Han, H.E. Logan, B. McElrath and L.-T. Wang, Phenomenology of the little Higgs model, Phys. Rev. D 67 (2003) 095004 [hep-ph/0301040] [INSPIRE]. 
[26] M. Schmaltz and D. Tucker-Smith, Little Higgs review, Ann. Rev. Nucl. Part. Sci. 55 (2005) 229 [hep-ph/0502182] [InSPIRE].

[27] H.-C. Cheng and I. Low, TeV symmetry and the little hierarchy problem, JHEP 09 (2003) 051 [hep-ph/0308199] [INSPIRE].

[28] H.-C. Cheng and I. Low, Little hierarchy, little Higgses and a little symmetry, JHEP 08 (2004) 061 [hep-ph/0405243] [INSPIRE].

[29] I. Low, T parity and the littlest Higgs, JHEP 10 (2004) 067 [hep-ph/0409025] [INSPIRE].

[30] J. Hubisz and P. Meade, Phenomenology of the littlest Higgs with T-parity, Phys. Rev. D 71 (2005) 035016 [hep-ph/0411264] [INSPIRE].

[31] ATLAS collaboration, Measurements of the properties of the Higgs-like boson in the four lepton decay channel with the ATLAS detector using $25 \mathrm{fb}^{-1}$ of proton-proton collision data, ATLAS-CONF-2013-013 (2013).

[32] ATLAS collaboration, Combined coupling measurements of the Higgs-like boson with the ATLAS detector using up to $25 \mathrm{fb}^{-1}$ of proton-proton collision data, ATLAS-CONF-2013-034 (2013).

[33] ATLAS collaboration, Study of the spin of the new boson with up to $25 \mathrm{fb}^{-1}$ of ATLAS data, ATLAS-CONF-2013-040 (2013).

[34] CMS collaboration, Combination of standard model Higgs boson searches and measurements of the properties of the new boson with a mass near $125 \mathrm{GeV}$, CMS-PAS-HIG-13-005.

[35] CMS collaboration, Measurement of the properties of a Higgs boson in the four-lepton final state, Phys. Rev. D 89 (2014) 092007 [arXiv: 1312.5353] [INSPIRE].

[36] S. Dawson et al., Working Group Report: Higgs Boson, arXiv:1310.8361 [INSPIRE].

[37] C. Englert et al., Precision Measurements of Higgs Couplings: Implications for New Physics Scales, J. Phys. G 41 (2014) 113001 [arXiv:1403.7191] [InSPIRE].

[38] A. Blondel et al., Report of the ICFA Beam Dynamics Workshop 'Accelerators for a Higgs Factory: Linear vs. Circular' (HF2012), arXiv:1302.3318 [INSPIRE].

[39] V.I. Telnov, Higgs factories, PoS (IHEP-LHC) 018) [arXiv:1307.3893] [INSPIRE].

[40] A. Kobakhidze, L. Wu and J. Yue, Anomalous Top-Higgs Couplings and Top Polarisation in Single Top and Higgs Associated Production at the LHC, arXiv:1406.1961 [INSPIRE].

[41] J. Cao, C. Han, L. Wu, J.M. Yang and M. Zhang, SUSY induced top quark FCNC decay $t \rightarrow$ ch after Run I of LHC, Eur. Phys. J. C 74 (2014) 3058 [arXiv:1404.1241] [INSPIRE].

[42] B. Yang, N. Liu and J. Han, Top quark flavor-changing neutral-current decay to a $125 \mathrm{GeV}$ Higgs boson in the littlest Higgs model with T parity, Phys. Rev. D 89 (2014) 034020 [INSPIRE].

[43] F. Simon, Higgs physics at future linear colliders - A case for precise vertexing, PoS (Vertex2013) 019 [arXiv: 1401.6302] [INSPIRE].

[44] N. Liu, J. Ren and B. Yang, Next-to-leading order QCD corrections to HZW $W^{ \pm}$production at 14 TeV LHC, Phys. Lett. B 731 (2014) 70 [arXiv:1310.6192] [InSPIRE].

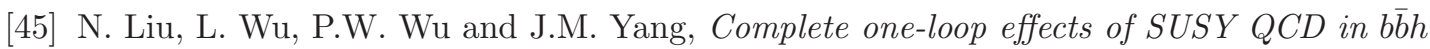
production at the LHC under current experimental constraints, JHEP 01 (2013) 161 [arXiv: 1208.3413] [INSPIRE]. 
[46] B. Yang, J. Han, S. Zhou and N. Liu, Single Higgs boson production at $e^{+} e^{-}$colliders in the Littlest Higgs Model with T-parity, J. Phys. G 41 (2014) 075009 [arXiv:1401.0289] [INSPIRE].

[47] S.L. Hu, N. Liu, J. Ren and L. Wu, Revisiting Associated Production of 125 GeV Higgs Boson with a Photon at a Higgs Factory, arXiv:1402.3050 [INSPIRE].

[48] N. Liu, J. Ren, L. Wu, P. Wu and J.M. Yang, Full one-loop electroweak corrections to $e^{+} e^{-} \rightarrow Z H \gamma$ at a Higgs factory, JHEP 04 (2014) 189 [arXiv:1311.6971] [INSPIRE].

[49] TLeP Design Study Working Group collaboration, M. Bicer et al., First Look at the Physics Case of TLEP, JHEP 01 (2014) 164 [arXiv:1308.6176] [INSPIRE].

[50] E.L. Berger, S.B. Giddings, H. Wang and H. Zhang, Higgs-flavon mixing and LHC phenomenology in a simplified model of broken flavor symmetry, arXiv:1406.6054 [INSPIRE].

[51] B. Gripaios, T. Müller, M.A. Parker and D. Sutherland, Search Strategies for Top Partners in Composite Higgs models, JHEP 08 (2014) 171 [arXiv: 1406.5957] [INSPIRE].

[52] A. Azatov, C. Grojean, A. Paul and E. Salvioni, Taming the off-shell Higgs boson, arXiv:1406.6338 [INSPIRE].

[53] D. Barducci et al., Model Independent Framework for Analysis of Scenarios with Multiple Heavy Extra Quarks, arXiv:1405.0737 [INSPIRE].

[54] J. Berger, J. Hubisz and M. Perelstein, A Fermionic Top Partner: Naturalness and the LHC, JHEP 07 (2012) 016 [arXiv: 1205.0013] [INSPIRE].

[55] C. Han, A. Kobakhidze, N. Liu, L. Wu and B. Yang, Constraining Top partner and Naturalness at the LHC and TLEP, arXiv:1405.1498 [INSPIRE].

[56] M. Koratzinos et al., TLEP: A High-Performance Circular $e^{+} e^{-}$Collider to Study the Higgs Boson, arXiv: 1305.6498 [INSPIRE].

[57] TLeP Design Study Working Group collaboration, M. Bicer et al., First Look at the Physics Case of TLEP, JHEP 01 (2014) 164 [arXiv:1308.6176] [INSPIRE].

[58] X.-F. Han, L. Wang, J.M. Yang and J. Zhu, Little Higgs theory confronted with the LHC Higgs data, Phys. Rev. D 87 (2013) 055004 [arXiv: 1301.0090] [INSPIRE].

[59] J. Reuter and M. Tonini, Can the 125 GeV Higgs be the Little Higgs?, JHEP 02 (2013) 077 [arXiv:1212.5930] [INSPIRE].

[60] J. Reuter, M. Tonini and M. de Vries, Little Higgs Model Limits from LHC - Input for Snowmass 2013, arXiv:1307.5010 [INSPIRE].

[61] J. Reuter, M. Tonini and M. de Vries, Littlest Higgs with T-parity: Status and Prospects, JHEP 02 (2014) 053 [arXiv: 1310.2918] [INSPIRE].

[62] C.-R. Chen, K. Tobe and C.-P. Yuan, Higgs boson production and decay in little Higgs models with T-parity, Phys. Lett. B 640 (2006) 263 [hep-ph/0602211] [INSPIRE].

[63] Particle Data Group collaboration, J. Beringer et al., Review of Particle Physics (RPP), Phys. Rev. D 86 (2012) 010001 [inSPIRE].

[64] J. Hubisz, P. Meade, A. Noble and M. Perelstein, Electroweak precision constraints on the littlest Higgs model with T parity, JHEP 01 (2006) 135 [hep-ph/0506042] [INSPIRE].

[65] M.E. Peskin and T. Takeuchi, Estimation of oblique electroweak corrections, Phys. Rev. D 46 (1992) 381 [inSPIRE]. 
[66] R. Barbieri, B. Bellazzini, V.S. Rychkov and A. Varagnolo, The Higgs boson from an extended symmetry, Phys. Rev. D 76 (2007) 115008 [arXiv:0706.0432] [INSPIRE].

[67] Z. Han and W. Skiba, Effective theory analysis of precision electroweak data, Phys. Rev. D 71 (2005) 075009 [hep-ph/0412166] [INSPIRE].

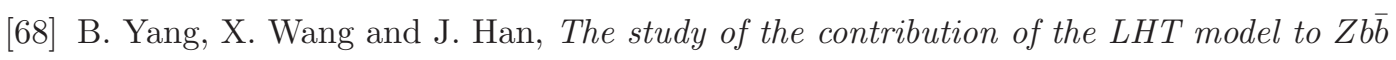
coupling, Nucl. Phys. B 847 (2011) 1 [arXiv:1103.2506] [INSPIRE].

[69] P. Bechtle, S. Heinemeyer, O. Stål, T. Stefaniak and G. Weiglein, HiggsSignals: Confronting arbitrary Higgs sectors with measurements at the Tevatron and the LHC,

Eur. Phys. J. C 74 (2014) 2711 [arXiv:1305.1933] [INSPIRE].

[70] P. Bechtle, O. Brein, S. Heinemeyer, G. Weiglein and K.E. Williams, HiggsBounds: Confronting Arbitrary Higgs Sectors with Exclusion Bounds from LEP and the Tevatron, Comput. Phys. Commun. 181 (2010) 138 [arXiv:0811.4169] [INSPIRE].

[71] P. Bechtle, S. Heinemeyer, O. Stål, T. Stefaniak and G. Weiglein, Probing the Standard Model with Higgs signal rates from the Tevatron, the $L H C$ and a future ILC, arXiv: 1403.1582 [INSPIRE].

[72] ATLAS collaboration, Search for heavy top-like quarks decaying to a Higgs boson and a top quark in the lepton plus jets final state in pp collisions at $\sqrt{s}=8 \mathrm{TeV}$ with the ATLAS detector, ATLAS-CONF-2013-018 (2013).

[73] S. Dawson et al., Working Group Report: Higgs Boson, arXiv:1310.8361 [INSPIRE]. 